\title{
Patients' Experiences in Different Models of Community Health Centers in Southern China
}

\author{
Harry H. X. Wang, $P b D^{1}$ \\ Samuel Y. S. Wong, MD, FCFPC ${ }^{1}$ \\ Martin C. S. Wong, MD, MPH \\ Xiao Lin Wei, MD, $\mathrm{PbD}^{1}$ \\ Jia Ji Wang, MD, MPH ${ }^{2}$ \\ Donald K. T. Li, FRACGP \\ Jin Ling Tang, $M D, P b D^{1}$ \\ Gemma Y. Gao, PbD ${ }^{1}$ \\ Sian M. Griffiths, FRCP, FFPH ${ }^{1}$ \\ ${ }^{1}$ School of Public Health and Primary Care, \\ Faculty of Medicine, The Chinese Univer- \\ sity of Hong Kong, Hong Kong \\ ${ }^{2}$ School of Public Health, Guangzhou \\ Medical University, Guangzhou, People's \\ Republic of China \\ ${ }^{3}$ Bauhinia Foundation Research Centre, \\ Hong Kong

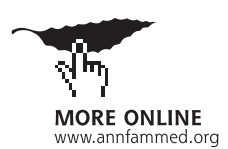

Conflicts of interest: authors report none.

\section{CORRESPONDING AUTHOR}

Samuel Y. S. Wong, MD, FCFPC

Division of Family Medicine and Primary Health Care

School of Public Health and Primary Care Prince of Wales Hospital, Faculty of Medicine

The Chinese University of Hong Kong

Shatin, NT, Hong Kong

yeungshanwong@cuhk.edu.hk

\begin{abstract}
PURPOSE Current health care reforms in China have an overall goal of strengthening primary care through the establishment and expansion of primary care networks based on community health centers (CHCs). Implementation in urban areas has led to the emergence of different models of ownership and management. The objective of this study was to evaluate the primary care experiences of patients in the Pearl River Delta as measured by the Primary Care Assessment Tool (PCAT) and the relationships with ownership and management in the 3 different models we describe.
\end{abstract}

METHODS This cross-sectional study was conducted on-site at CHCs in 3 cities within the Pearl River Delta, China, using a multistage cluster sampling method. A validated Mandarin Chinese version of the PCAT-Adult Edition (short version) was adopted to collect information from adult patients regarding their experiences with primary care sources. PCAT scores for individual primary care attributes and total primary care assessment scores were assessed with respect to sociodemographic characteristics, health characteristics, and health care service utilization across 3 primary care models.

RESULTS One thousand four hundred forty $(1,440)$ primary care patients responded to the survey, for an overall response rate of $86.1 \%$. Respondents gave government-owned and -managed CHCs the highest overall PCAT scores when compared with CHCs either managed by hospitals (95.18 vs 90.81 ; $P=.005)$ or owned by private and social entities (95.18 vs $90.69 ; P=.007)$ as a result of better first-contact care (better first-contact utilization) and coordination of care (better service coordination and information system). Factors that were positively and significantly associated with higher overall assessment scores included the presence of a chronic condition $(P<.001)$, having medical insurance $(P=.006)$, and a self-reported good health status $(P<.001)$.

CONCLUSIONS This study suggests that government-owned and -managed CHCs may be able to provide better first-contact care in terms of utilization and coordination of care, and may be better at solving the problem of underutilization of the CHCs as the first-contact point of care, one key problem facing the reforms in China.

Ann Fam Med 2013;517-526. doi:10.1370/afm.1545.

\section{INTRODUCTION}

I nternational experiences have shown that countries with a strong primary care-led health system have better population health and are able to achieve more equitable distribution of health. ${ }^{1}$ In the last 2 decades, the Chinese government has begun the process of establishing a community health service to achieve better population health..$^{2-5}$ In 2009, a health care reform plan was officially announced ${ }^{6}$ with a key component being the expansion of community health service facilities to serve as the first contact of care and gatekeepers to the entire health system. ${ }^{7}$ In urban areas, these facilities are organized around community health centers $(\mathrm{CHCs})^{8,9}$ and adhere to the national guidelines on the standard service provision set by the Ministry of Health. ${ }^{10}$ 
Health care staff in all CHCs are paid a fixed salary, plus a floating salary that is largely determined by the total income of the center. ${ }^{11}$ Preregistration is not necessary, and any patient can walk in to see a doctor after paying a fixed one-off registration fee set by the provincial Health Bureau. The payment for 1 visit also includes fees for examination, treatment, and the drug expenditure. For medically insured patients, the payment is shared between the government and the individuals. ${ }^{12,13}$ All $\mathrm{CHC}$ are required to have clinical physicians, public health doctors, and managerial and assistant health care staff. They also have on-site nurses, telephone access, and evening and weekend clinics. Most health care staff at $\mathrm{CHC}$ s regulated by the National Labour Act have similar working hours. ${ }^{14}$ Western and traditional Chinese medicine services are available in most $\mathrm{CHCs}{ }^{8}$

Because of the socioeconomic variations in different urban regions, the local implementation of $\mathrm{CHC}$ based primary care networks has led to the emergence of 3 categories of $\mathrm{CHC}$ models of ownership and management: (1) government-owned and -managed CHCs (G-CHCs), (2) government-owned and hospitalmanaged $\mathrm{CHCs}(\mathrm{H}-\mathrm{CHCs})$, and (3) privately owned and managed CHCs (P-CHCs). In 2008, 36.5\% of the $\mathrm{CHCs}$ were $\mathrm{G}-\mathrm{CHCs}$, $35.7 \%$ were $\mathrm{H}$-CHCs, and $27.8 \%$ were P-CHCs. ${ }^{5}$

G-CHCs, which are independent of the public hospital system, are part of the government sector and directly managed by the local government as nonprofit health care facilities..$^{15}$ The typical G-CHC is subject to the Separation of Revenue and Expenditure policy, ie, revenue generated by the G-CHC (including fees generated from medical treatment and drug sales) goes to the local government, and costs incurred by the G-CHC (including premises, equipment, facilities, and staff remuneration) are paid by the local government.

$\mathrm{H}$-CHCs are owned by the government but managed by the host hospital. They are not part of the government sector and are allowed to make a profit as financially self-sufficient institutions. The $\mathrm{H}-\mathrm{CHC}$ is usually regarded as a department within the hospital and typically functions as an outreach clinic. H-CHCs receive limited subsidies from the government through the host hospital, which further allocates funding to the $\mathrm{H}-\mathrm{CHC}$.

P-CHCs are owned and managed by private organizations and are independent of the government and hospital sectors. They do receive limited government subsidies, but they are financially self-sufficient. $\mathrm{P}$-CHCs provide primary care services in accordance with industry regulations ${ }^{8}$ (Figure 1).

One policy aspect relevant to government policy makers is the relationship between the ownership and management models of $\mathrm{CHCs}$ and the quality of care provided ${ }^{16}$ based on the key attributes of primary care. China's health care system is similar to that of the United States in that the system has been marketdriven; the result has been high overall health care spending without commensurately improved health

Figure 1. Three Emerging CHC Models for Primary Care Delivery in Urban Areas in China

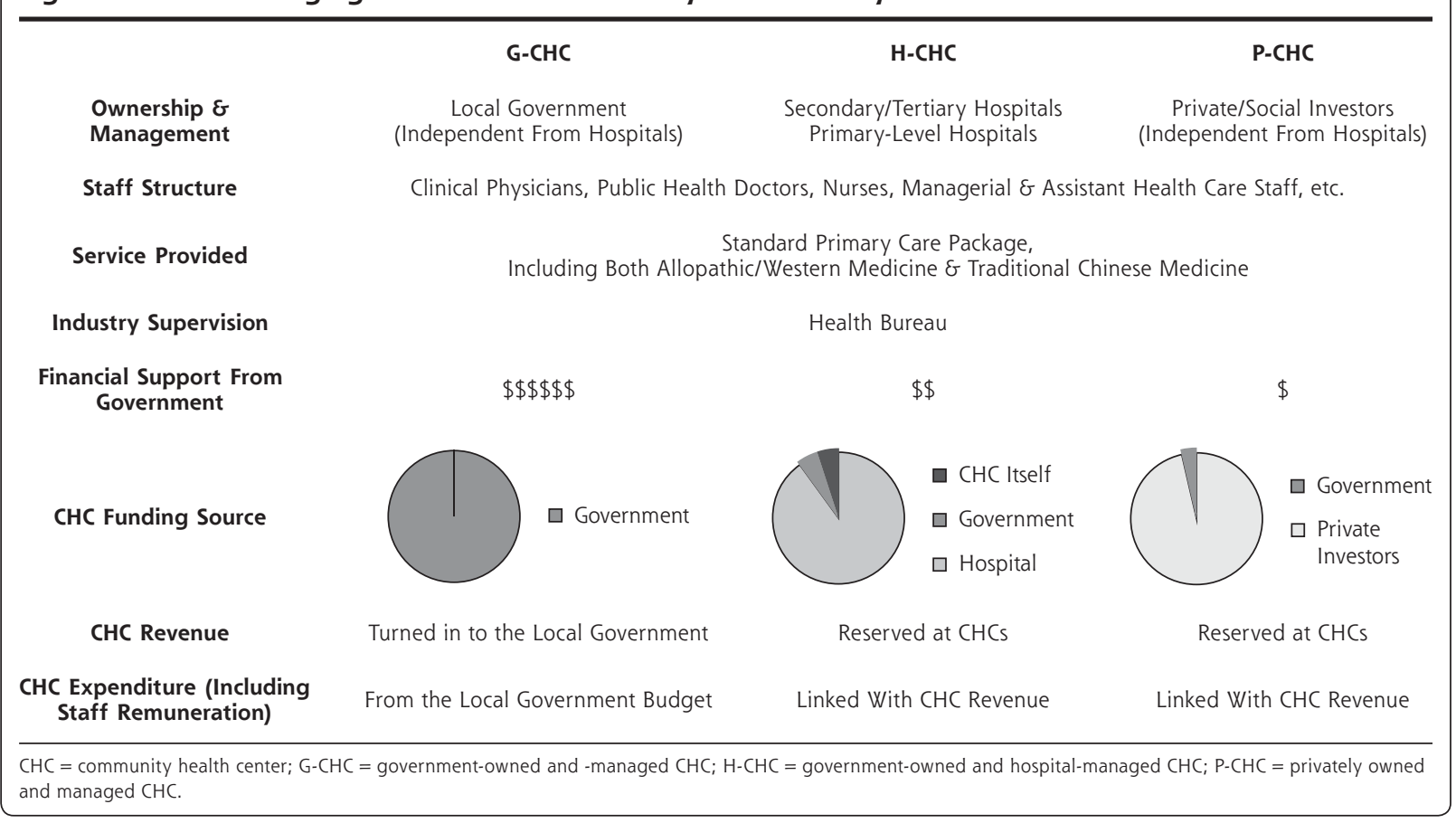


outcomes. In China fragmentation of care is common. Preventive, primary, and tertiary care are provided separately, leading to wastage of health care services. Coordination of care is often lacking among different health care providers, which compete for patients and hold onto them when they should be referred elsewhere. ${ }^{17}$ Our study examined the effect of various models of ownership and management on patients' experiences, especially in the primary care setting, in the anticipation that our findings would inform policy makers in both China and the United States.

\section{METHODS}

\section{Study Design}

This study was based on a large face-to-face patient survey conducted on-site at the CHCs in 6 cities within the Pearl River Delta, a geographic area in which the inhabitants' age and sex characteristics are similar to that of China's overall population. ${ }^{18}$ The region, considered to be a health care policy trendsetter, has well-established and mature primary care models. Three cities (city A, B, and C) were selected based on their differences and similarities. Each city has a different $\mathrm{CHC}$ organizational model under the current health system: city A has G-CHCs, city B has $\mathrm{H}-\mathrm{CHCs}$, and city $\mathrm{C}$ has P-CHCs. All 3 cities have similar municipal administrative levels, $\mathrm{CHC}$-to-population ratios, and rates of $\mathrm{CHC}$ outpatient visits. The selected CHCs also have similar staff structures and receive the same industry supervision, suggesting the study models are comparable.

A multistage cluster sampling method was adopted in each city (Supplemental Table, available at www.

annfammed.org/content/11/6/517/suppl/DC1). In

Hin the first stage, the districts were randomly selected as the primary sampling units. In the second stage, one neighborhood within each district was randomly selected as the secondary sampling units. In the third stage, one $\mathrm{CHC}$ within each neighborhood was randomly selected as the tertiary sampling units. Published literature ${ }^{19,20}$ shows that for analysis a maximum sample size of 300 per group was needed for a significance level of $5 \%$ with a power of $90 \%$. The final sample size for each model was 480 , and the design effect of this study was 3.40 , which was considered adequate to provide good statistical power.

\section{Survey Instrument}

A validated Mandarin Chinese version of the Primary Care Assessment Tool (PCAT)-Adult Edition (short version) was used to capture patients' experiences. ${ }^{19,21-23}$ Originally developed by Primary Care Policy Center at Johns Hopkins University, Baltimore, Maryland, the
PCAT has good cross-cultural adaptability for assessing primary care quality attributes from the consumer's viewpoint under different health care systems outside the United States. ${ }^{19,24-26}$ Validation of PCAT was conducted previously to ensure good reliability and validity for primary care assessment in China. ${ }^{22,23}$

The PCAT domains include the assessment of first-contact accessibility and utilization (first-contact domain), continuity of care (longitudinal domain), coordination of services and information system (coordination domain), comprehensiveness of service availability and provision (comprehensiveness domain), and community orientation and family centeredness (derivative domain). First-contact accessibility refers to whether patients are able to receive primary care whenever needed within a reasonable time, whereas first-contact utilization measures the extent to which the primary care provider performed a gatekeeper function. Service coordination measures the linking of health care visits between health system levels, whereas information system coordination measures the coordination of health records for patients. All items were scored according to a 4 -point Likert-type scale, with higher scores indicating a more positive experience. The total PCAT scores were calculated by summing all the values from each scale. ${ }^{21}$

\section{Study Population}

All patients aged 18 years or older who visited their health center on the day of recruitment were eligible to respond to the survey. A modified systematic random sampling was adopted to ensure that only those patients coming to the clinic for primary care were invited for inclusion. An adapted algorithm from the PCAT guideline was used to identify the respondents' regular source of primary care, including both frequent and less-frequent users.

\section{Interviewer Training}

Five postgraduate students attended 2 separate workshops to train as interviewers. Part of the training included independently coding the respondents' replies during practice sessions until an acceptable inter-rater reliability was achieved.

\section{Data Collection}

On-site data collection started in November 2010 for 6 months, and data were entered independently by 2 trained university students using EpiData software 3.1 (EpiData Association, Denmark). The study was approved by the Survey and Behavior Research Ethics Committee of The Chinese University of Hong Kong and the Ethics Committee of Guangzhou Medical University. 
Table 1. Characteristics of Study Respondents $(N=1,440)$

\begin{tabular}{|c|c|c|c|c|c|c|c|c|c|}
\hline \multirow[b]{2}{*}{ Variables } & \multicolumn{2}{|c|}{$\begin{array}{l}\text { All Patients } \\
(\mathrm{N}=1,440)\end{array}$} & \multicolumn{2}{|c|}{$\begin{array}{c}\text { City A } \\
\text { (G-CHC) } \\
(\mathrm{n}=480)\end{array}$} & \multicolumn{2}{|c|}{$\begin{array}{c}\text { City B } \\
\text { (H-CHC) } \\
(\mathrm{n}=480)\end{array}$} & \multicolumn{2}{|c|}{$\begin{array}{c}\text { City C } \\
\text { (P-CHC) } \\
(\mathrm{n}=480)\end{array}$} & \multirow{2}{*}{$\begin{array}{l}P \text { Value } \\
\left(x^{2}\right)\end{array}$} \\
\hline & No. & $\%$ & No. & $\%$ & No. & $\%$ & No. & $\%$ & \\
\hline \multicolumn{10}{|c|}{ Health care service utilization } \\
\hline \multicolumn{10}{|c|}{ Is there a CHC where you usually go } \\
\hline No & 193 & 13.4 & 46 & 9.6 & 55 & 11.5 & 92 & 19.2 & \\
\hline Yes & 1,247 & 86.6 & 434 & 90.4 & 425 & 88.5 & 388 & 80.8 & $<.001$ \\
\hline \multicolumn{10}{|c|}{ Frequency of $\mathrm{CHC}$ visits } \\
\hline$\leq 2$ & 494 & 34.6 & 197 & 41.1 & 171 & 35.6 & 126 & 26.8 & \\
\hline$\geq 3$ & 935 & 65.4 & 282 & 58.9 & 309 & 64.4 & 344 & 73.2 & $<.001$ \\
\hline \multicolumn{10}{|l|}{ Duration of $\mathrm{CHC}$ visits } \\
\hline$<1$ year & 740 & 58.2 & 305 & 74.2 & 212 & 49.3 & 223 & 51.7 & \\
\hline$\geq 1$ year & 532 & 41.8 & 106 & 25.8 & 218 & 50.7 & 208 & 48.3 & $<.001$ \\
\hline \multicolumn{10}{|c|}{ Hospital doctor visits after $\mathrm{CHC}$ visits } \\
\hline No & 656 & 45.6 & 194 & 40.4 & 255 & 53.1 & 207 & 43.1 & \\
\hline Yes & 784 & 54.4 & 286 & 59.6 & 225 & 46.9 & 273 & 56.9 & $<.001$ \\
\hline \multicolumn{10}{|c|}{ Presence of medical insurance } \\
\hline No & 346 & 24.0 & 122 & 25.4 & 124 & 25.8 & 100 & 20.9 & \\
\hline Yes & 1,093 & 76.0 & 358 & 74.6 & 356 & 74.2 & 379 & 79.1 & .138 \\
\hline \multicolumn{10}{|c|}{ Health characteristics } \\
\hline \multicolumn{10}{|c|}{ Presence of chronic medical condition } \\
\hline No & 902 & 62.6 & 313 & 65.2 & 301 & 62.7 & 288 & 60.0 & \\
\hline Yes & 538 & 37.4 & 167 & 34.8 & 179 & 37.3 & 192 & 40.0 & .249 \\
\hline \multicolumn{10}{|c|}{ Self-perceived health status } \\
\hline Fair or poor & 637 & 44.2 & 223 & 46.5 & 227 & 47.3 & 187 & 39.0 & \\
\hline Good or excellent & 803 & 55.8 & 257 & 53.5 & 253 & 52.7 & 293 & 61.0 & .017 \\
\hline \multicolumn{10}{|c|}{$\begin{array}{l}\text { Presence of self-perceived long-term } \\
\text { physical/mental problem }\end{array}$} \\
\hline No & 885 & 74.1 & 279 & 73.8 & 284 & 72.3 & 322 & 75.9 & \\
\hline Yes & 310 & 25.9 & 99 & 26.2 & 109 & 27.7 & 102 & 24.1 & $\begin{array}{l}.483 \\
\text { continues }\end{array}$ \\
\hline
\end{tabular}

\section{Data Analysis}

PCAT scores were assessed for individual primary care scales and overall scores. Multivariate analysis of variance and independent sample $t$ tests were performed to compare PCAT scores. Multiple linear regression analysis was performed to explore factors associated with overall PCAT scores. Multivariate analysis of covariance was conducted for comparison among different primary care models after Bonferroni-corrected adjustments were made for other covariates. The data were analyzed using PASW Statistics 18.0 (SPSS Inc).

\section{RESULTS}

A total of 1,659 primary care patients were approached for study inclusion, and 1,440 patients responded to the PCAT questionnaire. Excellent inter-rater reliability was achieved among the 5 interviewers (Fleiss' general- ized $\kappa=0.81 ; 95 \% \mathrm{CI}, 0.78-0.87 ; P<.001)$. The overall response rate was $86.1 \%$. No significant differences existed in the response rates at each site $(P=.419)$ and between respondents and nonrespondents in terms of age, sex, and medical insurance coverage. Patients differed by educational and income level in the 3 cities; however, there were no significant differences in terms of medical insurance coverage, chronic condition, self-perceived health problems, marital status, and sex distribution (Table 1).

After adjustments were made for sociodemographic characteristics, health care characteristics, and health utilization covariates, results showed that G-CHC respondents had the highest PCAT total scores when compared with $\mathrm{H}-\mathrm{CHC}(95.18$ vs $90.81 ; P=.005)$ and P-CHC (95.18 vs 90.69; $P=.007)$ respondents. When the individual domain scores of PCAT were compared among the 3 models of primary care service provision, 
Table 1. Characteristics of Study Respondents $(\mathrm{N}=1,440)$ (continued)

\begin{tabular}{|c|c|c|c|c|c|c|c|c|c|}
\hline \multirow[b]{2}{*}{ Variables } & \multicolumn{2}{|c|}{$\begin{array}{l}\text { All Patients } \\
(\mathrm{N}=1,440)\end{array}$} & \multicolumn{2}{|c|}{$\begin{array}{c}\text { City A } \\
\text { (G-CHC) } \\
(n=480)\end{array}$} & \multicolumn{2}{|c|}{$\begin{array}{c}\text { City B } \\
\text { (H-CHC) } \\
(n=480)\end{array}$} & \multicolumn{2}{|c|}{$\begin{array}{c}\text { City C } \\
\text { (P-CHC) } \\
(\mathrm{n}=480)\end{array}$} & \multirow{2}{*}{$\begin{array}{c}P \text { Value } \\
\left(\chi^{2}\right)\end{array}$} \\
\hline & No. & $\%$ & No. & $\%$ & No. & $\%$ & No. & $\%$ & \\
\hline \multicolumn{10}{|c|}{ Sociodemographic characteristics } \\
\hline Age, $y^{\text {a }}$ & \multicolumn{2}{|c|}{$43.4 \pm 17.1$} & \multicolumn{2}{|c|}{$37.2 \pm 13.2$} & \multicolumn{2}{|c|}{$47.2 \pm 17.7$} & \multicolumn{2}{|c|}{$41.0 \pm 14.4$} & \\
\hline$<55$ & 1,110 & 77.7 & 429 & 89.9 & 309 & 64.4 & 372 & 78.8 & \\
\hline$\geq 55$ & 319 & 22.3 & 48 & 10.1 & 171 & 35.6 & 100 & 21.2 & $<.001$ \\
\hline \multicolumn{10}{|l|}{ Sex, No. (\%) } \\
\hline Male & 601 & 41.8 & 218 & 45.4 & 185 & 38.5 & 198 & 41.3 & \\
\hline Female & 838 & 58.2 & 262 & 54.6 & 295 & 61.5 & 281 & 58.7 & .094 \\
\hline \multicolumn{10}{|l|}{ Marriage, No. (\%) } \\
\hline No & 241 & 16.7 & 81 & 16.9 & 66 & 13.8 & 94 & 19.6 & \\
\hline Yes & 1,198 & 83.3 & 399 & 83.1 & 414 & 86.3 & 385 & 80.4 & .051 \\
\hline \multicolumn{10}{|l|}{ Educational level, No. (\%) } \\
\hline Junior secondary or below & 851 & 59.1 & 391 & 81.5 & 298 & 62.1 & 162 & 33.8 & \\
\hline Senior secondary or above & 588 & 40.9 & 89 & 18.5 & 182 & 37.9 & 317 & 66.2 & $<.001$ \\
\hline \multicolumn{10}{|c|}{ Monthly household income per head } \\
\hline$<\because 2,000$ & 611 & 52.0 & 200 & 51.0 & 275 & 67.1 & 136 & 36.6 & \\
\hline$\geq ¥ 2,000$ & 563 & 48.0 & 192 & 49.0 & 135 & 32.9 & 236 & 63.4 & $<.001$ \\
\hline
\end{tabular}

Table 2. Comparison of Primary Care Assessment Scores in Different Primary Care Organizational Models

\begin{tabular}{|c|c|c|c|c|c|c|c|}
\hline \multirow{2}{*}{$\begin{array}{l}\text { Primary Care PCAT Scales } \\
\text { (Range of Values) }\end{array}$} & \multicolumn{3}{|c|}{ Score Mean (SE) } & \multicolumn{4}{|c|}{$P$ Value } \\
\hline & G-CHC & $\mathrm{H}-\mathrm{CHC}$ & P-CHC & G-H ${ }^{a}$ & $\mathrm{G}-\mathrm{Pb}^{\mathrm{b}}$ & H-Pc & Alld \\
\hline First contact: utilization (3-12) & $10.27(0.15)$ & $8.45(0.13)$ & $8.01(0.13)$ & $<.001$ & $<.001$ & .048 & $<.001$ \\
\hline First contact: accessibility (4-16) & $10.79(0.2)$ & $10.92(0.18)$ & $11.92(0.18)$ & $>.999$ & $<.001$ & $<.001$ & $<.001$ \\
\hline Continuity of care (4-16) & $11.42(0.17)$ & $11.30(0.16)$ & $11.69(0.16)$ & $>.999$ & .829 & .261 & .216 \\
\hline Coordination of services (4-16) & $12.22(0.23)$ & $11.27(0.24)$ & $11.40(0.22)$ & .022 & .048 & $>.999$ & .016 \\
\hline Coordination: information system (3-12) & $9.55(0.15)$ & $8.74(0.14)$ & $8.30(0.14)$ & .001 & $<.001$ & .068 & $<.001$ \\
\hline Comprehensiveness: services available (4-16) & $12.36(0.20)$ & $12.48(0.17)$ & $11.97(0.17)$ & $>.999$ & .475 & .111 & .095 \\
\hline Comprehensiveness: services provided $(5-20)$ & $14.17(0.23)$ & $14.09(0.21)$ & $13.64(0.21)$ & $>.999$ & .327 & .382 & .182 \\
\hline Family centeredness (3-12) & $9.05(0.18)$ & $8.86(0.16)$ & $8.74(0.16)$ & $>.999$ & .653 & $>.999$ & .467 \\
\hline Community orientation (3-12) & $7.20(0.18)$ & $7.03(0.16)$ & $6.94(0.16)$ & $>.999$ & .890 & $>.999$ & .579 \\
\hline Primary care total scores (33-132) & $95.18(0.99)$ & $90.81(0.90)$ & $90.69(0.92)$ & .005 & .007 & $>.999$ & .002 \\
\hline \multicolumn{8}{|c|}{$\begin{array}{l}\text { Note: higher values indicate a more positive experience. Scores are adjusted for duration of usual source of care, presence of medical insurance and chronic disease, } \\
\text { health status, sex, age, educational level, employment status, and household income. }\end{array}$} \\
\hline \multicolumn{8}{|c|}{$\begin{array}{l}\mathrm{CHC}=\text { community health center; } \mathrm{G}-\mathrm{CHC}=\text { government-owned } \mathrm{CHC} ; \mathrm{H}-\mathrm{CHC}=\text { hospital-operated } \mathrm{CHC} ; \mathrm{P}-\mathrm{CHC}=\text { privately-owned } \mathrm{CHC} ; \mathrm{PCAT}=\text { Primary Care Assessment } \\
\text { Tool; } \mathrm{SE}=\text { standard error. }\end{array}$} \\
\hline \multicolumn{8}{|l|}{ a $t$ Test comparing G-CHC with $\mathrm{H}-\mathrm{CHC}$. } \\
\hline \multicolumn{8}{|l|}{${ }^{\mathrm{b}} t$ Test comparing G-CHC with $\mathrm{P}-\mathrm{CHC}$. } \\
\hline \multicolumn{8}{|l|}{${ }^{c} t$ Test comparing $\mathrm{H}-\mathrm{CHC}$ with $\mathrm{P}-\mathrm{CHC}$. } \\
\hline
\end{tabular}

G-CHC respondents reported significantly higher PCAT scores for the first-contact (utilization) and coordination domains. The P-CHC respondents had the highest PCAT scores for the first-contact (accessibility) domain; however, $\mathrm{P}-\mathrm{CHC}$ respondents had relatively lower PCAT scores in nearly all other individual scales, especially for the first-contact (utilization) and coordination domains (Table 2).

Further analyses were conducted to explore the differences in PCAT scores based on medical insurance and chronic disease within each $\mathrm{CHC}$ model. We found that uninsured $\mathrm{H}-\mathrm{CHC}$ respondents had lower PCAT 
scores overall, suggesting a different primary care experience with this model (Table 3). Respondents who were chronically ill had significantly higher overall PCAT scores under G-CHCs (96.17 vs $92.17 ; P=.043)$ and $\mathrm{H}-\mathrm{CHCs}$ (94.06 vs $88.59 ; P=.010$ ) when compared with those who did not have chronic medical conditions. Respondents with chronic diseases under P-CHCs had significantly lower PCAT scores in terms of comprehensive service availability (11.34 vs 12.49 ; $P=.002)$ when compared with those who did not have chronic diseases in the same ownership and management model (Table 4).

There were no interaction effects found between $\mathrm{CHC}$ models and health utilization factors or socioeconomic factors, including income and educational level. Factors significantly associated with higher overall PCAT scores included a government-owned and -managed $\mathrm{CHC}(\mathrm{P}=.028$ for $\mathrm{H}-\mathrm{CHC}$; $\mathrm{P}=.009$ for $\mathrm{P}-\mathrm{CHC})$, regularly attending a $\mathrm{CHC}(P<.001)$, a most familiar doctor or nurse at the $\mathrm{CHC}(P<.001)$; medical insurance $(P=.006)$, a chronic medical condition $(P=.001)$, and good self-reported health status $(P<.001)$ (Table 5).

\section{DISCUSSION}

This study is the first to evaluate the relationship between organi-

zational and ownership models of CHCs and patients' experiences of primary care using an internationally developed and cross-culturally adapted tool in southern China. The PCAT, which focuses on patients' experience of, rather than satisfaction with, health care delivery, minimizes subjective bias that is due to sociodemographic variations and patient expectation. Previous studies have used PCAT to assess primary care under different health care systems and types of providers for patients with different sociodemographic attributes. ${ }^{19,20,24-34}$ This study adds to the evidence suggesting that the quality of primary care measured
Table 3. Comparison of Primary Care Assessment Scores in Different Primary Care Organizational Models by Medical Insurance

\begin{tabular}{|c|c|c|c|}
\hline \multirow[b]{2}{*}{ Primary Care Experience } & \multicolumn{2}{|c|}{ PCAT Score Mean (SE) ${ }^{a}$} & \multirow[b]{2}{*}{$P$ Value } \\
\hline & Uninsured & Insured & \\
\hline \multicolumn{4}{|l|}{ G-CHC (government owned and managed) } \\
\hline First contact: utilization & $10.21(0.26)$ & $10.02(0.14)$ & .526 \\
\hline First contact: accessibility & $10.82(0.39)$ & $10.63(0.20)$ & .668 \\
\hline Continuity of care & $11.29(0.36)$ & $11.29(0.18)$ & $>.999$ \\
\hline Coordination of services & $12.14(0.42)$ & $12.02(0.22)$ & .806 \\
\hline Coordination: information system & $9.12(0.26)$ & $9.37(0.13)$ & .405 \\
\hline Comprehensiveness: services available & $12.37(0.42)$ & $12.05(0.22)$ & .503 \\
\hline Comprehensiveness: services provided & $14.06(0.47)$ & $13.88(0.25)$ & .744 \\
\hline Family centeredness & $9.03(0.34)$ & $8.96(0.18)$ & .854 \\
\hline Community orientation & $7.04(0.33)$ & $7.02(0.17)$ & .962 \\
\hline Primary care total scores & $94.23(1.96)$ & $93.34(1.00)$ & .692 \\
\hline \multicolumn{4}{|l|}{$\begin{array}{l}\mathrm{H}-\mathrm{CHC} \text { (government owned and hospital } \\
\text { managed) }\end{array}$} \\
\hline First contact: utilization & $8.15(0.26)$ & $8.83(0.17)$ & .037 \\
\hline First contact: accessibility & $11.05(0.35)$ & $11.06(0.22)$ & .978 \\
\hline Continuity of care & $10.90(0.29)$ & $11.63(0.19)$ & .042 \\
\hline Coordination of services & $10.66(0.45)$ & $11.58(0.32)$ & .119 \\
\hline Coordination: information system & $8.05(0.26)$ & $9.11(0.17)$ & .001 \\
\hline Comprehensiveness: services available & $11.99(0.30)$ & $12.92(0.19)$ & .013 \\
\hline Comprehensiveness: services provided & $13.15(0.37)$ & $14.54(0.24)$ & .003 \\
\hline Family centeredness & $8.66(0.29)$ & $8.80(0.19)$ & .685 \\
\hline Community orientation & $6.45(0.29)$ & $7.29(0.18)$ & .018 \\
\hline Primary care total scores & $86.63(1.66)$ & $93.17(1.06)$ & .002 \\
\hline \multicolumn{4}{|l|}{ P-CHC (privately owned and managed) } \\
\hline First contact: utilization & $8.07(0.29)$ & $8.02(0.14)$ & .868 \\
\hline First contact: accessibility & $10.76(0.36)$ & $12.18(0.18)$ & $<.001$ \\
\hline Continuity of care & $11.49(0.34)$ & $11.76(0.17)$ & .474 \\
\hline Coordination of services & $11.80(0.43)$ & $11.54(0.24)$ & .607 \\
\hline Coordination: information system & $8.57(0.32)$ & $8.48(0.16)$ & .804 \\
\hline Comprehensiveness: services available & $12.25(0.35)$ & $11.97(0.17)$ & .470 \\
\hline Comprehensiveness: services provided & $14.30(0.45)$ & $13.76(0.22)$ & .279 \\
\hline Family centeredness & $8.79(0.36)$ & $8.95(0.18)$ & .685 \\
\hline Community orientation & $7.26(0.37)$ & $7.08(0.18)$ & .653 \\
\hline Primary care total scores & $91.79(2.01)$ & $92.02(0.99)$ & .922 \\
\hline \multicolumn{4}{|c|}{$\begin{array}{l}\mathrm{CHC}=\text { community health center; } \mathrm{G}-\mathrm{CHC}=\text { government-owned } \mathrm{CHC} ; \mathrm{H}-\mathrm{CHC}=\text { hospital operated } \mathrm{CHC} ; \\
\mathrm{P}-\mathrm{CHC}=\text { privately owned } \mathrm{CHC} ; \mathrm{PCAT}=\text { Primary Care Assessment Tool; } \mathrm{SE}=\text { standard error. }\end{array}$} \\
\hline
\end{tabular}

by patient experience is related to the ownership and management model of the CHCs.

The respondents gave G-CHCs higher overall PCAT scores as a result of better first-contact care (better first-contact utilization) and coordination of care. The higher score for first-contact utilization suggests that the better gatekeeping performance might be due to the more effective implementation of the reimbursement policy, which stipulates that patients shall be reimbursed only for health care expenditures occurring at a predesignated G-CHC, close to their living areas, ${ }^{35}$ thereby possibly reducing doc- 
Table 4. Comparison of Primary Care Assessment Scores in Different Primary Care Organizational Models by Chronic Disease

\begin{tabular}{|c|c|c|c|}
\hline \multirow[b]{2}{*}{ Primary Care Experience } & \multicolumn{2}{|c|}{ PCAT Score Mean (SE) ${ }^{a}$} & \multirow[b]{2}{*}{$P$ Value } \\
\hline & Presence & Absence & \\
\hline \multicolumn{4}{|l|}{ G-CHC (government owned and managed) } \\
\hline First contact: utilization & $10.06(0.21)$ & $10.06(0.15)$ & .988 \\
\hline First contact: accessibility & $10.89(0.32)$ & $10.56(0.22)$ & .409 \\
\hline Continuity of care & $11.70(0.29)$ & $11.07(0.20)$ & .084 \\
\hline Coordination of services & $12.62(0.34)$ & $11.76(0.24)$ & .049 \\
\hline Coordination: information system & $9.10(0.25)$ & $8.10(0.19)$ & .003 \\
\hline Comprehensiveness: services available & $12.43(0.35)$ & $11.98(0.24)$ & .304 \\
\hline Comprehensiveness: services provided & $14.32(0.39)$ & $13.71(0.27)$ & .218 \\
\hline Family centeredness & $9.21(0.27)$ & $8.85(0.19)$ & .300 \\
\hline Community orientation & $7.07(0.28)$ & $7.00(0.19)$ & .850 \\
\hline Primary care total scores & $96.17(1.56)$ & $92.17(1.10)$ & .043 \\
\hline \multicolumn{4}{|l|}{$\begin{array}{l}\text { H-CHC (government owned and hospital } \\
\text { managed) }\end{array}$} \\
\hline First contact: utilization & $8.88(0.22)$ & $8.39(0.21)$ & .145 \\
\hline First contact: accessibility & $11.25(0.29)$ & $10.89(0.27)$ & .418 \\
\hline Continuity of care & $11.89(0.25)$ & $10.98(0.23)$ & .015 \\
\hline Coordination of services & $11.37(0.44)$ & $11.17(0.36)$ & .763 \\
\hline Coordination: information system & $8.99(0.22)$ & $8.60(0.20)$ & .236 \\
\hline Comprehensiveness: services available & $12.66(0.26)$ & $12.61(0.24)$ & .886 \\
\hline Comprehensiveness: services provided & $14.64(0.31)$ & $13.62(0.30)$ & .032 \\
\hline Family centeredness & $9.17(0.25)$ & $8.40(0.23)$ & .036 \\
\hline Community orientation & $7.39(0.24)$ & $6.71(0.23)$ & .055 \\
\hline Primary care total scores & $94.06(1.41)$ & $88.59(1.30)$ & .010 \\
\hline \multicolumn{4}{|l|}{ P-CHC (privately owned and managed) } \\
\hline First contact: utilization & $8.26(0.22)$ & $7.88(0.17)$ & .202 \\
\hline First contact: accessibility & $11.48(0.28)$ & $12.17(0.22)$ & .065 \\
\hline Continuity of care & $11.33(0.26)$ & $11.95(0.20)$ & .078 \\
\hline Coordination of services & $11.67(0.37)$ & $11.57(0.28)$ & .840 \\
\hline Coordination: information system & $9.57(0.21)$ & $9.18(0.15)$ & .137 \\
\hline Comprehensiveness: services available & $11.34(0.27)$ & $12.49(0.21)$ & .002 \\
\hline Comprehensiveness: services provided & $13.96(0.34)$ & $13.80(0.27)$ & .729 \\
\hline Family centeredness & $9.61(0.28)$ & $8.48(0.22)$ & .003 \\
\hline Community orientation & $7.50(0.29)$ & $6.86(0.22)$ & .101 \\
\hline Primary care total scores & $93.00(1.55)$ & $91.30(1.21)$ & .418 \\
\hline \multicolumn{4}{|c|}{$\begin{array}{l}\mathrm{CHC}=\text { community health center; } \mathrm{G}-\mathrm{CHC}=\text { government-owned } \mathrm{CHC} ; \mathrm{H}-\mathrm{CHC}=\text { hospital-operated } \mathrm{CHC} \text {; } \\
\mathrm{P}-\mathrm{CHC}=\text { privately owned } \mathrm{CHC} ; \mathrm{PCAT}=\text { Primary Care Assessment Tool; } \mathrm{SE}=\text { standard error. }\end{array}$} \\
\hline $\begin{array}{l}\text { a Primary care assessment scores adjusted for } \\
\text { health status, sex, age, educational level, emp }\end{array}$ & f usual source & $\begin{array}{l}\text { re, presence of } \mathrm{cl} \\
\text { ld income. }\end{array}$ & c disease, \\
\hline
\end{tabular}

Previous studies conducted in the United States ${ }^{37,38}$ have shown that government-funded and -regulated $\mathrm{CHCs}$ were able to deliver a higher quality of primary care when compared with hospital outpatient clinics or private doctors' offices in terms of better service coordination, comprehensiveness, and community orientation. In a recent Canadian study ${ }^{39}$ that compared several organizational models and outcomes for chronic disease management, government-funded $\mathrm{CHCs}$ were associated with better chronic disease care when compared with other organizational models. It is worthy to note that the key differences among these government-funded and -managed primary care facilities are that the CHCs in the United States and Canada are thirdsector organizations operated under community governance, ${ }^{40}$ whereas the G-CHCs in China are part of the government sector and managed directly by the local governmental departments. ${ }^{15}$

Under the $\mathrm{H}-\mathrm{CHC}$ and P-CHC models, the role of local government in providing primary care is limited. In these models, primary care delivery is governed by hospitals and private investors, which are financially self-sufficient, for-profit organizations. ${ }^{41}$ Our results show that $\mathrm{H}-\mathrm{CHC}$ respondents who were not medically insured had significantly lower overall and individual

tor shopping. The higher score of G-CHCs in the coordination domain could be explained by the community-wide multisectoral initiatives and joint efforts made at different governmental levels to integrate health service connections under the government's strategic plan. ${ }^{36}$ The results also showed that a higher proportion of all respondents had follow-up visits at hospitals and they gave better overall PCAT scores to G-CHCs in the coordination domain for those who were chronically ill, suggesting a more cohesive linkage of health care services between different levels of health system. domain PCAT scores. When comparing medical insurance coverage between $\mathrm{H}$-CHC survey respondents and the general population, ${ }^{42} \mathrm{H}$-CHC respondents without medical insurance coverage reported less health service utilization, suggesting $\mathrm{H}-\mathrm{CHC}$ might be less utilized by vulnerable patients. In addition, the lower PCAT scores for service coordination and fewer subsequent visits by patients at hospitals might indicate a weaker linkage between $\mathrm{H}-\mathrm{CHC}$ and different levels of health system.

International studies have shown inconsistency in the quality of primary care provided by the private sector. ${ }^{19,43}$ Although China's P-CHCs have less-rigid admin- 


\section{Table 5. Multiple Linear Regression Analysis on Primary Care} Assessment Score

\begin{tabular}{|c|c|c|c|}
\hline $\begin{array}{l}\text { Dependent Variable: } \\
\text { Primary Care Achievement } \\
\text { (Total Score) }\end{array}$ & $\beta(95 \% \mathrm{Cl})$ & SE & $P$ Value \\
\hline Intercept & $76.096(71.186-81.006)$ & 2.502 & $<.001$ \\
\hline \multicolumn{4}{|l|}{$\begin{array}{l}\text { Organizational models (primary care } \\
\text { settings) }\end{array}$} \\
\hline $\mathrm{G}-\mathrm{CHC}$ & $\ldots$ & $\ldots$ & $\ldots$ \\
\hline $\mathrm{H}-\mathrm{CHC}$ & $-3.019(-5.714$ to -0.324$)$ & 1.373 & .028 \\
\hline $\mathrm{P}-\mathrm{CHC}$ & $-3.631(-6.346$ to -0.917$)$ & 1.383 & .009 \\
\hline \multicolumn{4}{|l|}{ Health care utilization } \\
\hline Regularly attending the $\mathrm{CHC}$ & 7.828 (4.932 to 10.725$)$ & 1.476 & $<.001$ \\
\hline Most familiar doctor/nurse at the $\mathrm{CHC}$ & 6.686 (4.609 to 8.764$)$ & 1.059 & $<.001$ \\
\hline Frequency of $\mathrm{CHC}$ visits $\geq 3$ times & $1.570(-0.581$ to 3.720$)$ & 1.096 & .152 \\
\hline Duration of $\mathrm{CHC}$ visits $\geq 1 \mathrm{y}$ & $1.707(-0.391$ to 3.805$)$ & 1.069 & .111 \\
\hline Have hospital visits after $\mathrm{CHC}$ visits & $0.818(-1.522$ to 3.157$)$ & 1.192 & .493 \\
\hline Presence of medical insurance & 2.783 (0.818 to 4.749$)$ & 1.001 & .006 \\
\hline \multicolumn{4}{|l|}{ Health characteristics } \\
\hline Presence of chronic disease & 3.644 (1.399 to 5.888$)$ & 1.144 & .001 \\
\hline $\begin{array}{l}\text { Self-perceived health status good or } \\
\text { excellent }\end{array}$ & $4.436(2.387$ to 6.484$)$ & 1.044 & $<.001$ \\
\hline \multicolumn{4}{|l|}{ Sociodemographic characteristics } \\
\hline Female & $-0.540(-2.487$ to 1.407$)$ & 0.992 & .587 \\
\hline Age $\geq 55$ y & $-1.691(-4.673$ to 1.290$)$ & 1.519 & .266 \\
\hline $\begin{array}{l}\text { Educational level senior secondary } \\
\text { or higher }\end{array}$ & $2.292(-0.009$ to 4.593$)$ & 1.173 & .051 \\
\hline Employed & $1.811(-0.751$ to 4.373$)$ & 1.305 & .166 \\
\hline $\begin{array}{l}\text { Monthly income per head of house- } \\
\text { hold } \geq ¥ 2,000\end{array}$ & $-0.499(-2.543$ to 1.546$)$ & 1.042 & .632 \\
\hline
\end{tabular}

covererage, and a self-reported good health status. Patient's sociodemographic factors were not sensitive to overall PCAT scores shown in the study. A study conducted in Hong Kong ${ }^{19}$ previously reported that higher PCAT scores were found in patients with chronic conditions and those with medical insurance. The national agenda under China's current health care reform requires local commitment from CHCs to cope with noncommunicable chronic diseases ${ }^{48-50}$ so that chronically ill patients might receive more attention. Meanwhile, progress toward the expansion of medical insurance coverage $e^{12,13}$ might enable patients to have better primary care experiences.

This large cross-sectional study has a number of limitations. First, many unmeasured confounders potentially exist between $\mathrm{CHC}$ ownership and management and PCAT scores, and a patient self-report survey restricted inclusion of questions relating to physician information, which was not accounted for in this istrative and bureaucratic structures and may be more responsive to local needs, ${ }^{44,45}$ the profit-driven nature and the poorer coordination of care with other health services might result in a poorer primary care experience, in particular where the care is less incentivized. ${ }^{46}$ Our study showed that respondents gave P-CHCs lower overall PCAT scores, especially in the coordination domain. One of the explanations could be that the P-CHC model might lack incentives to refer patients to other care services provided by other health organizations that compete for patient enrolment, ${ }^{44}$ although we cannot confirm this explanation based on our current findings. Lower PCAT scores given by P-CHC respondents with chronic diseases (in comprehensiveness domain) and by respondents with lower household incomes (in first-contact utilization and service availability domain) (figure available from authors upon request) might be due to the lack of government subsidies to provide more comprehensive services, as $\mathrm{P}$-CHCs receive the least financial support from the local government. ${ }^{47}$

Factors positively associated with better primary care experiences among all respondents also included having a chronic condition, having medical insurance study. Second, there were differences in the patients' characteristics, although factors previously identified to be significantly associated with PCAT scores were all controlled for. Third, we have not used symptoms or disease-specific outcomes; instead, we used a validated tool measuring only the key attributes of primary care. Thus, our study is related more to the process of care.

\section{Implications for Policy Making and Future Perspectives}

We showed that patients who identified governmentowned and -managed CHCs (G-CHCs) as their regular source of primary care reported better primary care experiences, especially with respect to the first-contact (utilization) and coordination of care. Our findings imply that G-CHCs may be able to better solve the problem of underutilization of primary care organizations as the first-contact point of care, which is one of the key problems facing China's health care reforms. ${ }^{51}$ Further work should examine whether the better primary care attributes observed in the G-CHCs are related to other unexplored factors and might provide more causal evidence for improvement of primary care. 
To read or post commentaries in response to this article, see it online at www.annfammed.org/content/11/6/517.

Key words: primary health care; primary care assessment tool; community health center; ownership and management model

Submitted September 9, 2012; submitted, revised, February 17, 2013; accepted March 12, 2013.

Funding support: This study was funded by the Bauhinia Foundation Research Centre Limited, Hong Kong.

Prior presentations: The validation of the survey instrument was previously presented at the 19th WONCA Asia Pacific Regional Conference, 24-27 May 2012, Jeju, Korea: SY02-2/ SY18/AF0344; and at the 18th WONCA Asia Pacific Regional Conference and 10th WONCA World Rural Health Conference, 20-24 February 2011, Cebu, Philippines: ABS-OP0156.

Acknowledgments: We wish to acknowledge the support of the local health care managers in Guangdong Province, who made this study run smoothly. The excellent work of the student helpers from both Guangzhou Medical University and The Chinese University of Hong Kong ensured the high quality of the completed study.

\section{References}

1. Starfield B, Shi LY, Macinko J. Contribution of primary care to health systems and health. Milbank Q. 2005;83(3):457-502.

2. Decision concerning public health reform and development. http:// www.moh.gov.cn/wsb/pM30115/200804/18540.shtml. People's Republic of China: State Council; 1997.

3. Guidance on Development of Community Health Services in the Cities. No.10 document. http://www.gov.cn/zwgk/2006-02/23/content_208882.htm. People's Republic of China: State Council; 2006.

4. Chen Z, Gao Q. Moving towards universal medical services with Chinese characteristics. http://www.gov.cn/gzdt/2008-01/02/content_848758.htm. People's Republic of China: Ministry of Health; 2008.

5. Centre for Health Statistics and Information. Research on Health Services of Primary Health Care Facilities in China, 2008. People's Republic of China: Ministry of Health; 2008.

6. Chen Z. Launch of the health-care reform plan in China. Lancet. 2009;373(9672):1322-1324.

7. Yip W, Hsiao W. China's health care reform: a tentative assessment. China Econ Rev. 2009;20(4):613-619.

8. Guidelines and Opinions on the Establishment of Community Health Service Facilities in Urban Areas. No.96 document. http://www.gov. cn/zwgk/2006-09/04/content_377067.htm. People's Republic of China: CCP Central Committee; 2006.

9. Wang HHX, Wang JJ. Developing primary care in China. In: Griffiths SM, Tang JL, Yeoh EK, eds. Routledge Handbook of Global Public Health in Asia. Oxford: Routledge; 2014.

10. The announcement of the construction standard of community health centres and community health stations in urban areas. No. 240 document. http://www.gov.cn/zwgk/2006-08/10/content_359200.htm. People's Republic of China: Ministry of Health; 2006.

11. Staff remuneration. No. 3 document. http://kjs.mof.gov.cn/zhuantilanmu/kuaijizhuanzeshishi/200806/t20080618_46239.html. People's Republic of China: Ministry of Finance; 2006.

12. Implementation guidance on the establishment of basic medical insurance system for urban residents. No. 75 document. http:// zwgk.gd.gov.cn/006939748/200909/t20090915_9492.html. People's Republic of China: Guangdong government; 2007.
13. Guiding Opinions of the State Council about the Pilot Urban Resident Basic Medical Insurance. No. 20 document. http://www.gov. cn/zwgk/2007-07/24/content_695118.htm. People's Republic of China: State Counsil; 2007.

14. National Labour Act. http://www.gov.cn/banshi/2005-08/05/content_20688.htm. People's Republic of China: The Central People's Government; 2005.

15. Accelerating the community health service development in Dongguan. No. 96 document. http://www.dg.gov.cn/business/htmlfiles/ cndg/s28614/201109/412906.htm. People's Republic of China: Dongguan government; 2007.

16. Crampton P, Starfield B. A case for government ownership of primary care services in New Zealand: weighing the arguments. International journal of health services : planning, administration, evaluation 2004:34(4):709-27.

17. Yip W, Hsiao WC. Market watch - The Chinese health system at a crossroads. Health Aff (Millwood). 2008;27(2):460-468.

18. The Sixth National Population Census. National Bureau of Statistics of China., 2011.

19. Wong SYS, Kung K, Griffiths SM, et al. Comparison of primary care experiences among adults in general outpatient clinics and private general practice clinics in Hong Kong. BMC Public Health. 2010;10:397.

20. Shi L, Starfield B, Xu J, Politzer R, Regan J. Primary care quality: community health center and health maintenance organization. South Med J. 2003;96(8):787-795.

21. Shi LY, Starfield B, Xu JH. Validating the adult primary care assessment tool. J Fam Pract. 2001;50(2):161w-175w.

22. Wang HHX, Wong MCS, Wong SYS, et al. Development of a Chinese version of Primary Care Assessment Tool for evaluation of primary care delivery in the Mainland China: a pilot study (ABS-OP0156). Oral presentation at the 18th WONCA Asia Pacific Regional Conference and 10th WONCA World Rural Health Conference. Cebu, Philippines, 2011.

23. Wang HHX, Wong MCS, Wong SYS, et al. Primary Care Assessment Tool: tests of reliability and validity of the Mandarin Chinese version in mainland China (SYOZ-2/ SY18/AF0344). Oral presentation at the 19th WONCA Asia Pacific Regional Conference. Jeju, Korea, 2012.

24. Lee JH, Choi YJ, Sung NJ, et al; Korean Primary Care Research Group. Development of the Korean primary care assessment toolmeasuring user experience: tests of data quality and measurement performance. Int J Qual Health Care. 2009;21(2):103-111.

25. Pongpirul K, Starfield B, Srivanichakorn S, Pannarunothai S. Policy characteristics facilitating primary health care in Thailand: a pilot study in transitional country. Int J Equity Health. 2009;8:8.

26. Tsai J, Shi L, Yu WL, Lebrun LA. Usual source of care and the quality of medical care experiences: a cross-sectional survey of patients from a Taiwanese community. Med Care. 2010;48(7):628-634.

27. Starfield B, Cassady C, Nanda J, Forrest CB, Berk R. Consumer experiences and provider perceptions of the quality of primary care: implications for managed care. J Fam Pract. 1998;46(3):216-226.

28. Haggerty JL, Pineault R, Beaulieu MD, et al. Practice features associated with patient-reported accessibility, continuity, and coordination of primary health care. Ann Fam Med. 2008;6(2):116-123.

29. Tourigny A, Aubin M, Haggerty J, et al. Patients' perceptions of the quality of care after primary care reform: Family medicine groups in Quebec. Can Fam Physician. 2010;56(7):e273-e282.

30. Berra S, Rocha KB, Rodriguez-Sanz M, et al. Properties of a short questionnaire for assessing Primary Care experiences for children in a population survey. BMC Public Health. 2011;11:285.

31. Rocha KB, Rodríguez-Sanz M, Pasarín MI, Berra S, Gotsens M, Borrell C. Assessment of primary care in health surveys: a population perspective. Eur J Public Health. 2012;22(1):14-19. 
32. Harzheim E, Duncan BB, Stein AT, et al. Quality and effectiveness of different approaches to primary care delivery in Brazil. BMC Health Serv Res. 2006;6:156.

33. Macinko J, Almeida C, de Sá PK. A rapid assessment methodology for the evaluation of primary care organization and performance in Brazil. Health Policy Plan. 2007;22(3):167-177.

34. Tsai J, Shi L, Yu WL, Hung LM, Lebrun LA. Physician specialty and the quality of medical care experiences in the context of the Taiwan national health insurance system. J Am Board Fam Med. 2010;23(3): 402-412.

35. Announcement on establishing social basic medical insurance system in Dongguan. No. 51 document. http://www.dg.gov.cn/business $/ \mathrm{htm} /$ files/cndg/s2569/201109/409774.htm. People's Republic of China: Dongguan government; 2008.

36. Recent major strategic plan for pharmaceutical and healthcare system reform in Dongguan (2009-2011). No. 71 document. http:// zwgk.gd.gov.cn/007330010/201105/t20110513_89112.html. People's Republic of China: Dongguan government; 2010.

37. Starfield B, Powe NR, Weiner JR, et al. Costs vs quality in different types of primary care settings. JAMA. 1994;272(24):1903-1908.

38. Rittenhouse DR, Robinson JC. Improving quality in Medicaid: the use of care management processes for chronic illness and preventive care. Med Care. 2006;44(1):47-54.

39. Russell GM, Dahrouge S, Hogg W, Geneau R, Muldoon L, Tuna M. Managing chronic disease in ontario primary care: the impact of organizational factors. Ann Fam Med. 2009;7(4):309-318.

40. Crampton P. The ownership elephant: ownership and communitygovernance in primary care. N Z Med J. 2005;118(1222):U1663.

41. Yang HJ, Zhou ZH, Li FJ, Wang JJ. SWOT analysis on the community health service models of "hospital-conducted and hospital-managed model" and "unified management model" [in Chinese]. Chinese Health Serv Manage. 2011;28(03):172-175.
42. National Census. 2010 Main Social and Economic Indicators: Statistics Bureau of Guangdong Province Government., 2010.

43. Care Quality Commission. Great Britain. Parliament. House of Commons. Care Quality Commission Annual Report and Accounts for the Period 1 April 2010 to 31 March 2011. London: Stationery Office, 2011.

44. Liu C, Wang J. The SWOT analysis of privately-owned community health service facilities in China [in Chinese]. J Community Med. 2009;7(01):64-66.

45. Chi Y, Yao L, Zhang L. Advantages and challenges of privatelyowned community health service facilities [in Chinese]. Chinese Health Econ. 2007;298(12):10-12.

46. Zhu H. Zhang I, Yao L. Analysis of policies and management systems of privately-owned community health service facilities [in Chinese]. Chinese Health Econo. 2007;297(11):43-45.

47. Zhao K, Zhang YC, Yao HX. Comparative analysis on service delivery of community health service institutions of different ownerships [in Chinese]. Chinese Gen Pract. 2010;13(8A):2430-2432.

48. Yang GH, Kong LZ, Zhao WH, et al. Emergence of chronic noncommunicable diseases in China. Lancet. 2008;372(9650):1697-1705.

49. Healthy China Plan 2020. http://www.gov.cn/gzdt/2008-01/07/content_851780.htm. Ministry of Health; 2008.

50. What can be learned from China's health system? Lancet. 2012;379 (9818):777-77.

51. Yang YS, Yang D. Community health service centers in China, not always trusted by the populations they serve? China Econ Rev. 2009; 20(4):620-624. 